\title{
SPHERICALLY SYMMETRIC FUNCTIONS WITH A CONVEX SECOND DERIVATIVE AND APPLICATIONS TO EXTREMAL PROBABILISTIC PROBLEMS
}

\author{
IOSIF PINELIS
}

\begin{abstract}
We describe the class of all functions $\varphi:[0, \infty) \rightarrow \mathbb{R}$ for which the second derivative $g_{\varphi}^{\prime \prime}(x ; y, y)$ of the spherically symmetric function $g_{\varphi}(x):=\varphi(|x|)$ in the direction of $y$ is convex in $x$, where $x$ and $y$ are vectors in a Hilbert space $H$ and $|\cdot|$ is the norm in $H$. Applications to extremal probabilistic problems are given.
\end{abstract}

Mathematics subject classification (2000): Primary: 26B25, 60E15; Secondary: 26D10, 34A40, 52A 40, 52A41.

Key words and phrases: Spherically symmetric (spherically invariant, rotation-invariant, elliptically contoured) functions; convexity; convex second derivative; extremal probabilistic problems; probabilistic inequalities; Khinchine inequality; generalized moments; generalized moment comparison inequalities; random multilinear (multi-affine) forms; Rademacher chaos; Hilbert space; sums of independent random variables; differential inequalities.

\section{REFERENCES}

[1] P. BiLlingsLey, Convergence of Probability Measures, (1968) Wiley, New York.

[2] D. C. COX AND J. H. B. KeMPERMAN, Sharp bounds on the absolute moments of a sum of two i. i. d. random variables. Ann. Probab. 11 (1983), 765-771.

[3] M. L. Eaton, A note on symmetric Bernoulli random variables, Ann. Math. Statist. 41 (1970), 1223-1226.

[4] M. L. EATON, A probability inequality for linear combinations of bounded random variables, Ann. Statist. 2 (1974), 609-614.

[5] R. KanNan And C. K. KRUEGer, Advanced Analysis on the Real Line (1996), Springer, New York.

[6] I. F. PINELIS, Some estimates for distributions of sums of independent random variables, Theory Probab. Appl., 29 (1984), 186-187.

[7] I. PINELIS, Extremal probabilistic problems and Hotelling's $T^{2}$ test under a symmetry condition, Ann. Statist., 22 (1994), 357-368.

[8] I. F. PINELIS AND S. A. UTEV, Estimates of moments of sums of independent random variables, Theory Probab. Appl., 29 (1984), 574-577.

[9] I. F. PINELIS AND S. A. UTEV, Sharp exponential estimates for sums of independent random variables, Theory Probab. Appl., 34 (1989), 340-346.

[10] YU. V. Prokhorov, Extremal problems in limit theorems. (Russian), Proceedings of the 6th All-Union Conference on Probability Theory and Mathematical Statistics (1962), 77-84.

[11] H. P. Rosenthal, On the span in $L^{p}$ of sequences of independent random variables, Proceedings of the Sixth Berkeley Symposium on Mathematical Statistics and Probability, 2 (1972), 149-167.

[12] S. A. UTEv, Extremal problems in moment inequalities. (Russian) Limit theorems of probability theory, 56-75, 175, Trudy Inst. Mat., 5 "Nauka" Sibirsk. Otdel., Novosibirsk, 1985.

[13] P. WhitTLE, Bounds for the moments of linear and quadratic forms in independent variables, Teor. Verojatnost. i Primenen. 5 (1960), 331-335. 